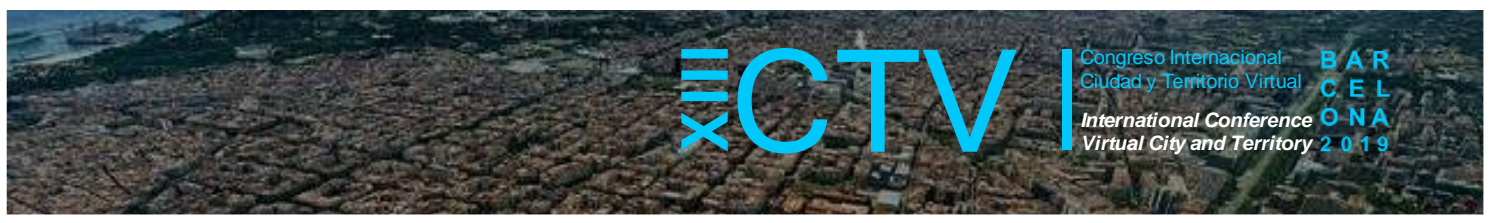

\title{
MIDIENDO LOS PROCESOS DE GENTRIFICACIÓN EN BARCELONA Y MADRID: UNA PROPUESTA METODOLÓGICA
}

\author{
López-Gay, Antonio ${ }^{1 *}$; Sales-Favà, Joan ${ }^{2}$; Solana, Miguel ${ }^{3}$; Fernández, Ana ${ }^{4}$; Peralta, Andrés ${ }^{5}$
}

Remisión inicial: 2019-06-16; Remisión definitiva: 2019-10-07; Publicación: 2019-12-21

Citación: López-Gay, A. et al. (2019). Midiendo los procesos de gentrificación en Barcelona y Madrid: una propuesta metodológica. En XIII CTV 2019 Proceedings: XIII International Conference on Virtual City and Territory: "Challenges and paradigms of the contemporary city": UPC, Barcelona, October 2-4,2019. Barcelona:CPSV,2019,p.8680. E-ISSN2604-6512.DOI http://dx.doi.org/10.5821/ctv.8680

\section{Resumen}

Las ciudades occidentales están experimentando intensas transformaciones en su configuración socio-espacial. Por un lado, los espacios exclusivos se expanden rápidamente desde los centros metropolitanos, de la mano de una inmigración cada vez más cualificada y de un contexto de resurgimiento de los espacios centrales vinculado a la localización de nuevas actividades productivas y a la concentración de enclaves culturales, creativos y de innovación. Por el otro, recientes estudios muestran que la población con menos ingresos se ve desplazada y concentrada en espacios más periféricos, con peor acceso a todo tipo de servicios. Como resultado, los diferentes grupos sociales se estarían alejando territorialmente. En la línea del primer proceso, el de la gentrificación es el ejemplo más local y visible para definir la transformación de un barrio. El elemento común entre las muchas definiciones interdisciplinares que se le han dado a los procesos de gentrificación, es el del reemplazo de la población, entendido como una substitución sociodemográfica consecuencia de la llegada a un territorio de población de clase media-alta que contribuye a la expulsión de los residentes previos, normalmente con menos recursos. Pese a que este proceso fue conceptualizado hace más de cinco décadas, el abordaje cuantitativo del proceso de substitución y desplazamiento de la población ha tenido menor recorrido que su abordaje teórico. Académicos e instituciones públicas de ámbito local y regional han intentado medir, desde lo cuantitativo, la gentrificación. Estas aproximaciones han buscado categorizar los barrios según la intensidad del proceso utilizando variables poblacionales, geográficas y/o urbanísticas. Pese a todo, la diversidad de las fuentes de cada país, y la dificultad de profundizar en los procesos de substitución a través de los datos, explican la falta de una metodología común y estándar para medir estos procesos a nivel internacional. En el caso español, las limitaciones a nivel de detalle geográfico del último censo de población, así como su lejanía en el tiempo, se realizó en 2011, han contribuido al vacío que tenemos sobre el enfoque cuantitativo a los procesos de gentrificación más recientes.

En este artículo proponemos un ejercicio metodológico y conceptual con el fin de calcular un indicador compuesto que mida la intensidad de los procesos de transformación sociodemográfica vinculados a la gentrificación que han experimentado los barrios de Barcelona y Madrid desde 2011 hasta 2017. El indicador incluye siete dimensiones teóricas asociadas a diferentes ejes de transformación socioeconómica del barrio: (1) rejuvenecimiento de la población; (2) cambios en la naturaleza de la población, entendida como el crecimiento de aquella procedente de los países con un Índice de Desarrollo Humano más alto; (3) cambios en las estructuras de los hogares/valores, asociado al crecimiento de los hogares unipersonales de adultos; (4) atracción a población con estudios universitarios; (5) substitución de la población, entendida como la pérdida de población sin estudios universitarios por efecto de la migración y los cambios de residencia; (6) velocidad del cambio, que introduce la capacidad transformadora de los flujos migratorios y residenciales de alterar la composición de la población; (7) transformaciones en el mercado de la vivienda, medido a través del aumento del precio. Una de las innovaciones de este indicador es la diversa naturaleza de las fuentes utilizadas, ya que contempla la utilización de fuentes demográficas de stock (el padrón continuo) y de flujos (los registros de altas y bajas del padrón acompañados de una variable socioeconómica como el nivel de instrucción), características de los hogares y sobre el mercado de la vivienda.

\footnotetext{
1 Investigador TALENT, Departament de Geografia de la Universitat Autònoma de Barcelona y Centre d'Estudis Demogràfics, https://orcid.org/0000-0001-8892-2816; 2 Investigador, Centre d'Estudis Demogràfics, https://orcid.org/0000-0002-2346-9552; ${ }^{3}$ Profesor titular, Departament de Geografia de la Universitat Autònoma de Barcelona, https://orcid.org/0000-0002-0811-1990; ${ }^{4}$ Técnica en salud pública, Servei de Salut Comunitaria / Grup d'Habitatge i Salut, Agència de Salut Pública de Barcelona, https://orcid.org/0000-0002-0333-1345 y ${ }^{5}$ Investigador Predoctoral, Grup d'Habitatge i Salut, Agència de Salut Pública de Barcelona y Grup de Recerca en Desigualtats Socials de la Salut (GREDS-EMCONET), https://orcid.org/0000-0002-7617-108X * Correo de contacto: tlopez@ced.uab.cat
} 


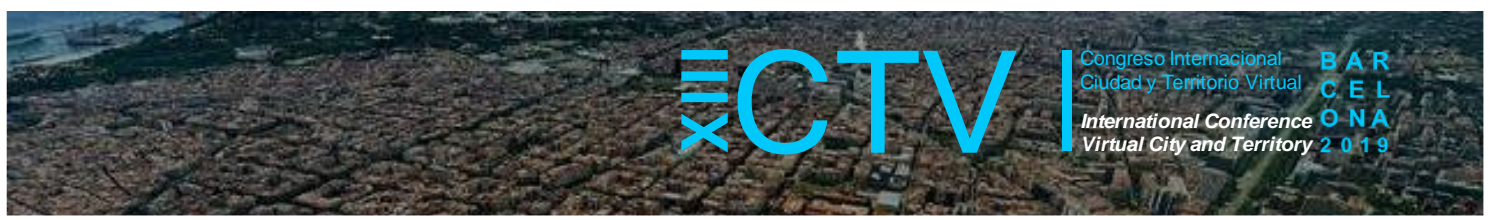

El resultado final del ejercicio no sólo nos ha permitido identificar qué barrios de Barcelona y Madrid han vivido procesos de gentrificación en los últimos años, sino también, y quizás más interesante, medir con qué intensidad. El indicador compuesto final se ha calculado en función de unos parámetros comunes que permiten comparar los índices de los barrios de una y otra ciudad, además de trazar los principales ejes de expansión de los procesos de gentrificación.

\begin{abstract}
Western cities are witnessing first-hand significant transformations in their socio-spatial configuration. On the one hand, exclusive areas are expanding rapidly from the metropolitan centers due to an increasingly qualified immigration and the resurgence of central spaces linked to the location of new productive activities and the concentration of cultural, creative and innovative enclaves. On the other hand, recent studies show that low-income residents are displaced and concentrated in the most peripheral and deprived spaces, with less access to all kinds of services. As a result, different social groups would be moving away territorially. In line with the first process, gentrification is the most local and visible example to define the transformation of a neighborhood. The common element among the many interdisciplinary definitions that have been given to gentrification processes is that of population replacement, understood as a sociodemographic substitution resulting from the arrival of upper-middle class population that contributes to the expulsion of previous residents, usually with fewer resources. Although this process was conceptualized more than five decades ago, the quantitative approach to the process of substitution and displacement of the population has been less developed than its theoretical approach. Academics and public institutions at local and regional level have tried to measure gentrification from a quantitative perspective. These approaches have sought to categorize neighborhoods according to the intensity of the process using population, geographic and / or urban variables. However, the diversity of sources in each country and the complexity of measuring displacement and substitution processes through data, explain the lack of a common and standard methodology to measure these processes at an international level. In the Spanish case, the geographic limitations of the last population census, as well as its distance in time, was carried out in 2011, have contributed to the lack of a quantitative approach to the most recent gentrification processes.
\end{abstract}

In this paper, we propose a methodological and conceptual exercise to calculate a composite indicator measuring the intensity of the sociodemographic transformation processes linked to gentrification that the neighborhoods of Barcelona and Madrid have experienced from 2011 to 2017. The indicator includes seven theoretical dimensions associated with different axes of neighborhood socioeconomic transformation: (1) population rejuvenation; (2) changes in the origin of the population, understood as the growth of population born in countries with a high Human Development Index; (3) changes in the family arrangements/values, associated with the growth of single-person adult households; (4) attraction to population with university degree; (5) population substitution, understood as the loss of population without university studies due to the effect of migration and changes of residence; (6) speed of change, which introduces the transformative capacity of migratory and residential flows to alter the composition of the population; (7) transformations in the housing market, measured through price increases. One of the innovations of the indicator is the diversity of the data sources we have used, since it includes stock data (Population Register), flow data (migration and residential flows, including a socioeconomic variable such as level of education), characteristics of households and housing market indicators.

The final values of the indicator have not only allowed us to identify which neighborhoods of Barcelona and Madrid have experienced gentrification processes in recent years, but also, and probably more interestingly, to measure its intensity. The indicator has been calculated based on common parameters, granting us not only to compare the indexes of the neighborhoods of both cities, but also to trace the main axes of expansion of the gentrification processes.

Palabras Clave: cambio urbano; gentrificación; indicador de gentrificación; España

Key words: urban change; gentrification; gentrification index; Spain

\title{
1. Introducción. Desigualdades sociales y procesos de segregación y gentrificación en la ciudad contemporánea
}

La creciente polarización social tiene su reflejo sobre el territorio, y más concretamente sobre los espacios urbanos. Ya en 1991 Saskia Sassen en su estudio sobre la globalización y el surgimiento de las ciudades globales apuntó a los procesos de polarización que se estaban produciendo en las principales ciudades del mundo debido a los cambios en la actividad económica y a las características de los flujos migratorios que se dirigían a estas ciudades: 


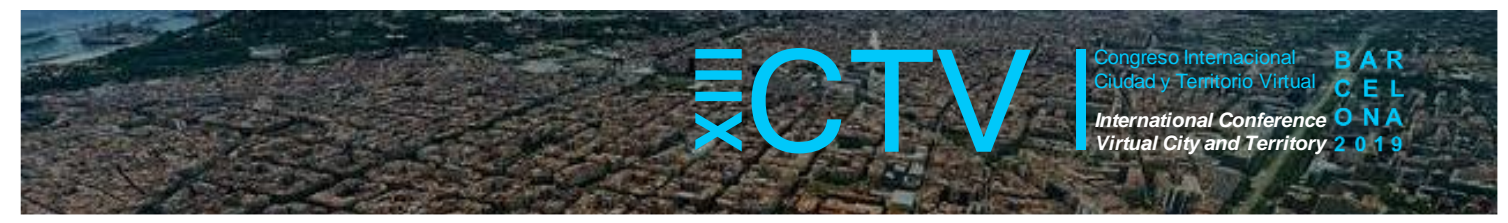

migrantes que ocupan los segmentos más precarizados del mercado de trabajo urbano junto con la recepción de migrantes, o residentes ocasionales, con elevados niveles socioprofesionales y con elevados niveles de renta. Bauman (1998) se referirá a esta última población como las élites globales, que disfrutan entre otras ventajas de una capacidad ilimitada de movilidad. La preocupación por el impacto que tienen estos flujos migratorios y la creciente desigualdad social han dado lugar a numerosos estudios sobre los procesos de gentrificación urbanos (Lees, 2012; Lees et al., 2008). Muchos de estos, además, remarcan su carácter global y subrayan la conveniencia de estudiar dichos procesos, con sus particularidades sociales e históricas, en los centros urbanos del sur global (Albet y Benach, 2018; Atkinson y Bridge, 2005; Lees et al., 2016).

La literatura internacional más reciente subraya el alejamiento territorial de los diferentes grupos sociales en las ciudades líderes occidentales como consecuencia de dos grandes procesos. Por un lado, los espacios exclusivos se expanden rápidamente desde los centros metropolitanos, de la mano de una inmigración cada vez más cualificada y con realidades laborales más globales (Sassen, 2018), y de un contexto de resurgimiento de los espacios centrales vinculado a la localización de nuevas actividades productivas (Hutton, 2009) y a la concentración de enclaves culturales, creativos y de innovación (Musterd, 2006). Por el otro, recientes estudios muestran que la población más vulnerable en muchas ciudades del mundo se ve desplazada y concentrada en espacios más periféricos, con peor acceso a todo tipo de servicios (Albet y Benach, 2018; Bailey y Minton. 2018; Hochstenbach y Musterd, 2017). En la línea del primer proceso, la gentrificación es la expresión más local y visible para definir la transformación de un barrio. El elemento común entre las muchas definiciones interdisciplinares que se le han dado a los procesos de gentrificación, es el del reemplazo de la población, entendido como una substitución sociodemográfica consecuencia de la llegada a un territorio de población de clase media-alta que contribuye a la expulsión de los residentes previos, normalmente con menos recursos (Glass, 1964; Gale, 1985).

En muchas ciudades, las dinámicas del mercado de la vivienda junto con el empobrecimiento de partes importantes de la población conducen a procesos de desplazamiento de la misma. Especialmente en aquellos países donde son más patentes dichas desigualdades sociales y territoriales, como es el caso de España (Martínez García, 2013; Sarasa et al., 2017), y donde no se han articulado políticas claras y prolongadas temporalmente que hayan permitido asegurar el acceso del conjunto de la población a la vivienda (Donat, 2018). Diversos estudios que tienen como ámbito de estudio España y sus principales centros urbanos indican el incremento de la desigualdad socioeconómica y la creciente segregación residencial (LópezGay, 2016a, 2016b, 2018; Porcel et al, 2018; Rubiales, 2016; Rubiales et al., 2012; Sorando y Ardura, 2018; Sorando y Leal, 2019).

Es en dicho marco de globalización, aumento de la polarización social, dificultad en el acceso a la vivienda y transformación urbana que se enmarca este artículo. En este contexto se propone un ejercicio metodológico y conceptual con el fin de desarrollar un indicador compuesto que mida la intensidad de los procesos de transformación sociodemográfica asociados a la gentrificación que han experimentado los barrios de Barcelona y Madrid entre 2011 y 2017.

\section{El canario en la mina: ¿cómo detectar los procesos de gentrificación urbana?}

Pese a que la gentrificación fue definida hace más de cinco décadas, el abordaje cuantitativo del proceso de substitución y desplazamiento de la población ha tenido menor recorrido que su 


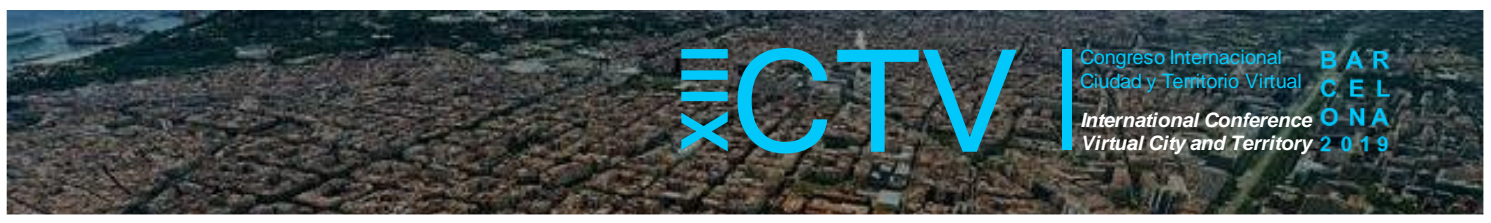

tratamiento teórico. Académicos e instituciones públicas de ámbito local y regional han intentado medir, desde lo cuantitativo, la gentrificación. Estas aproximaciones han buscado categorizar los barrios según la intensidad del proceso utilizando variables poblacionales, geográficas y/o urbanísticas. Pese a todo, la diversidad de las fuentes de cada país, y la dificultad de profundizar en los procesos de substitución a través de los datos, explican la falta de una metodología común y estándar para medir estos procesos a nivel internacional. En el caso español, las limitaciones a nivel de detalle geográfico del último censo de población, así como su lejanía en el tiempo, se realizó en 2011, han contribuido al vacío que tenemos sobre el enfoque cuantitativo a los procesos de gentrificación más recientes.

La creación de un sistema de índices e indicadores que permitan detectar los procesos de emergencia de gentrificación y los lugares afectados se extienden por diversas ciudades del mundo. La revisión de diferentes propuestas permite observar la necesidad de adaptarlas, como se ha indicado anteriormente, a las características y contexto histórico que presenta cada ciudad. Y también, a la disponibilidad de la información sociodemográfica existente. Por lo tanto, no se encuentran réplicas de un mismo indicador entre diferentes contextos, si bien en algunos casos se detecta la filiación de ciudades/áreas urbanas que utilizan un modelo que presenta características comunes. En este caso nos fijaremos exclusivamente en las propuestas de tipo cuantitativo que intentan capturar el proceso de gentrificación, si bien desde aproximaciones cualitativas también se pueden hacer contribuciones complementarias y valiosas al estudio del fenómeno (Bondi, 1999; Atkinson, 2000).

Tres son los aspectos que queremos reseñar en este análisis, preliminar y que no pretende ser exhaustivo, que se ha realizado de un conjunto de indicadores sobre gentrificación.

Primero, es conveniente destacar la heterogeneidad de instituciones y organizaciones que han fomentado y elaborado dichos indicadores. En algunos casos son las propias autoridades locales las que han impulsado dichos índices, como en Los Angeles Index of Neighborhood Change, financiado por la fundación Bloomberg Philantropies. En la mayor parte de casos, sin embargo, se tratan de indicadores elaborados por grupos de investigación académica. En éstos, no es extraño encontrar la participación de organizaciones de la sociedad civil que luchan contra los procesos de expulsión de la población de sus barrios. Sería el caso de los análisis llevados a cabo en diferentes regiones urbanas de Estados Unidos bajo el paraguas del Urban Displacement Project: Los Ángeles, San Francisco, Portland, Nueva York o Boston. La Tabla 1 muestra las variables recogidas, según tipología, en cada uno de los proyectos. También muestra autores relevantes que recalcan como importantes algunas de estas variables para caracterizar los procesos de gentrificación.

Tabla 1. Aspectos más relevantes utilizados en los indicadores para caracterizar la gentrificación

\begin{tabular}{lc}
\hline Concepto & Autores \\
\hline Naturaleza de la población & (Atkinson, 2000; I-team, 2016; Bianco et al., 2018*; Verma et al., 2018*) \\
\hline Condición socioeconómica & (Freeman, 2005; Sorando y Ardura, 2016; Ding et al., 2016; Martin y Beck, 2018) \\
\hline Hogares y cambios de valores & (Hall et al., 1997; Atkinson, 2000; Ogden y Schnoebelen, 2005; Atkinson et al., \\
2011) \\
\hline Estructura por edades & (Atkinson, 2000; Atkinson et al., 2011; Moos et al., 2018; Riera, 2018) \\
\hline Alta movilidad y rotación & (Newman y Wyly, 2006; Atkinson et al., 2011; Rérat y Lees, 2011) \\
\hline $\begin{array}{l}\text { Características de la vivienda } \\
\text { Localización y características } \\
\text { de la zona }\end{array}$ & (Freeman, 2005; I-team, 2016; Verma et al., 2018*; Riera, 2018) \\
\hline uente: elaboración propia. Nota* *Urban Displacement Project
\end{tabular}

Fuente: elaboración propia. Nota: *Urban Displacement Project 


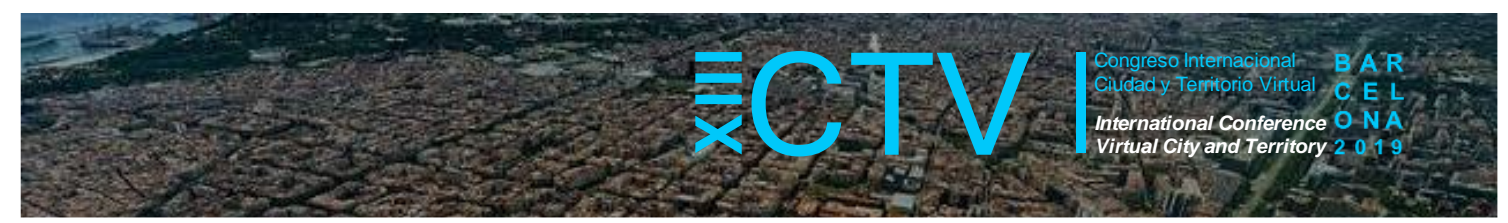

En segundo lugar, los aspectos que se suelen tener en cuenta a la hora de medir los procesos de gentrificación y expulsión de la población son diversos. Muchos indicadores pretenden captar el cambio en la composición sociodemográfica de la población, a través de diferentes dimensiones, sobre todo las relacionadas con el origen/etnia, nivel de estudios, relación con la actividad, tipo de ocupación y estructura por edad. Para analizar esa transformación, la mayor parte de estudios comparan las características de la población entre dos momentos determinados (análisis de stocks). También existen ejemplos de trabajos que introducen la composición de la población y de los hogares que entran y abandonan las unidades analizadas (análisis de flujos), aunque son menos frecuentes porque pocas veces se dispone de esta información. Es frecuente, también, que se introduzcan variables que capturan las características de la vivienda o la localización del barrio (cf. Tabla 1).

El tercer elemento que queremos destacar es el objetivo de dichos indicadores. Podríamos separar entre los que miden el riesgo o la potencialidad de un área a ser gentrificada y los que miden la intensidad del fenómeno en una zona específica. Tal y como el estudio de Atkinson et al. (2011) propone, la gentrificación se concibe como un proceso y, por tanto, los indicadores buscan descartar en principio aquellas áreas que quedan fuera de dicho proceso, ya sea porque se encuentran con una población con niveles de renta y socio-profesionales altos o muy altos, barrios donde se haya o no producido gentrificación previamente; ya sea porque se trata de áreas que por las características urbanas y la localización no muestran indicios de gentrificación, y se constituyen en zonas de concentración de pobreza y precariedad. Los indicadores, por tanto, tienen como objetivo determinar los espacios donde es posible detectar un riesgo potencial de gentrificación según las características socio-urbanísticas del barrio, ya que reúnen una serie de condiciones que los convierten en potencialmente atractivos para la inversión de grupos inmobiliarios, procesos de renovación urbana y residencial y expulsión de la población. Una vez identificados, utilizando más variables de naturaleza sociodemográfica, se analiza si el proceso ya se está produciendo, y con qué niveles de intensidad (Atkinson et al, 2011; Ding et al, 2016; Freeman, 2005; Martin y Beck, 2018).

En España se han realizado recientemente varias propuestas para calcular un índice de gentrificación. En este caso, Barcelona y Madrid son los ámbitos territoriales que se han tenido normalmente en cuenta debido a sus dimensiones, su importancia económica y social, y las tensiones que refleja el mercado de la vivienda fruto de los procesos de incremento de la desigualdad económica y los procesos de gentrificación (Antón-Alonso et al., 2018; Sorando y Ardura, 2018; Riera, 2018). Estos trabajos corren en paralelo con una mayor tradición de estudios en que se ha intentado medir los procesos de segregación social territorial (Rubiales et al, 2012; Sorando y Leal, 2019), y que también proponen la creación de indicadores cuantitativos que permitan detectar el proceso y su evolución e intensidad. En este sentido, la gentrificación aparece como un subproducto de la caracterización de la ciudad en diferentes niveles de segregación y no un como un tema de análisis propio. La naturaleza del indicador que proponemos en este trabajo nos permite captar la incidencia del fenómeno en un marco temporal específico.

Además, tenemos la capacidad de dilucidar entre los barrios elitizados y no elitizados al inicio del periodo de estudio. A diferencia de los indicadores que analizan el potencial gentrificador de una zona, nuestra propuesta cuantifica el nivel de desarrollo de un fenómeno en proceso o ya acabado. Por esta razón la mayoría de variables que se utilizan tienen que ver con los protagonistas de la gentrificación, la población (tanto la desplazada como la que desplaza). 


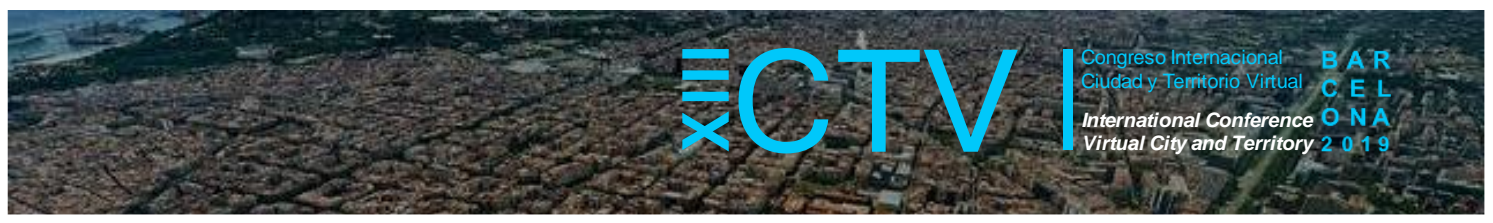

\section{Metodología: propuesta para la creación de un indicador de gentrificación comparable en las ciudades españolas}

El objetivo de este trabajo es generar un índice de gentrificación, de naturaleza esencialmente sociodemográfica, que permita identificar qué barrios de los municipios de Barcelona y Madrid han experimentado procesos asociados a los de la gentrificación entre 2011 y 2017 . El índice que proponemos está determinado por la disponibilidad de variables comparables entre las dos ciudades y recoge muchas de las transformaciones que han sido apuntadas en trabajos que han abordado el tema o que han sido incluidas en otros ejercicios similares.

Entre las innovaciones de este indicador está la diversa naturaleza de los datos y el uso pionero de alguna de las fuentes de información utilizadas. El indicador de gentrificación se alimenta de cuatro grandes fuentes de datos: el padrón continuo del Instituto Nacional de Estadística, la lectura del padrón municipal a nivel de hogar, la base de microdatos con todos los flujos migratorios y residenciales y los informes sobre el mercado de la vivienda de idealista a nivel de barrio, diseminados a partir de los propios ayuntamientos. Por motivos de representatividad, hemos agrupado a todos los barrios en los que en 2011 residían menos de 3.000 habitantes con los barrios limítrofes más parecidos en términos de renta. Así, hemos pasado de la división original de 204 barrios (73 en Barcelona y 131 en Madrid), a una de 190 barrios (67 en Barcelona y 123 en Madrid). La unidad de análisis, el barrio, es plenamente comparable entre las dos ciudades, ya que su población media ronda las 25.000 personas en ambos casos. Nuestro indicador compuesto combina siete dimensiones conceptuales diferentes que están estrechamente asociadas con los cambios que se experimentan en los sectores que la literatura ha descrito como gentrificados.

- Dimensión 1: Rejuvenecimiento de la población. Los espacios que viven procesos de gentrificación experimentan un crecimiento de la población adulta-joven (Moss et al., 2019). En el caso español, no obstante, es muy importante tener en cuenta el efecto del paso de las generaciones llenas (aprox. 1960-1975) por la vida adulta. Como éstas no se distribuyen aleatoriamente en los diferentes barrios de ambas ciudades, en este ejercicio no mediremos el rejuvenecimiento de la población comparando un mismo grupo de edad en dos cortes transversales, sino que seguiremos la evolución de las cohortes nacidas entre 1972 y 1997 (que cumplirán 45 años y 20 años en 2017, respectivamente) en los dos momentos analizados: 1-I-2011 y 1-I-2017. Aquellos barrios en los que más ha crecido la proporción de población nacida entre 1972 y 1997 puntúan más alto en el indicador de gentrificación.

Dimensión 1 (rejuvenecimiento): $\frac{\text { Pob72_97 } 2017_{20}}{\text { PobTOTAL }_{2017}}-\frac{\text { Pob72_97 } 2011_{\text {PobTOTAL }} 2011}{\text { PobT }_{10}}$

- Dimensión 2: Naturaleza de la población. El origen de la población ha sido incluido frecuentemente en los indicadores de gentrificación. En el caso español, y a diferencia del contexto anglosajón, apostamos por medir la variación de la población procedente de países con ingresos altos, en vez de los bajos. Dos motivos principales lo justifican. Por un lado, la migración internacional de baja cualificación en España es relativamente reciente, con lo que el abandono de determinados barrios no tiene por qué denotar un desplazamiento/expulsión, sino que puede obedecer al propio proceso de asentamiento en la metrópolis (Bayona y López-Gay, 2011). Además, en el periodo de crisis económica la evolución de este colectivo ha estado especialmente afectada por la migración de retorno o la re-emigración. Por el otro lado, la literatura apunta a que los procesos de gentrificación en el contexto del sur de Europa están 


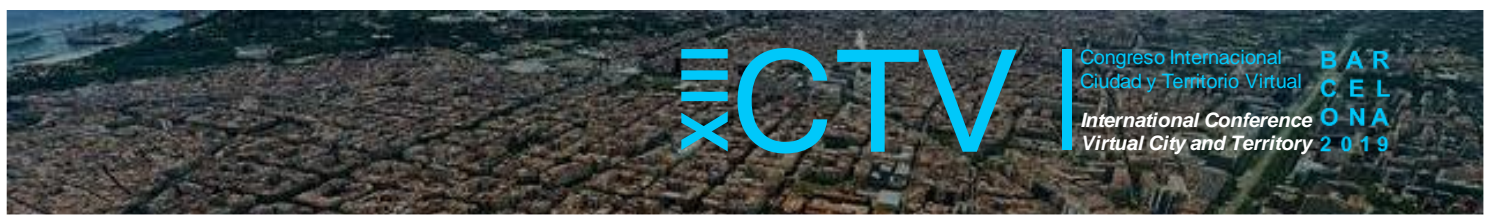

cada vez más ligados a la llegada de población internacional muy cualificada, que además es cada vez más móvil (López-Gay, 2016a). Como el padrón no nos ofrece ninguna variable socioeconómica según la naturaleza de la población, proponemos aproximarnos a esta dimensión utilizando el Índice de Desarrollo Humano (IDH) de la ONU. Aquellos barrios en los que más ha crecido (entre 1-I-2011 y 1-I-2017) la proporción de población nacida en países con un IDH superior a 0,825 puntúan más alto en el indicador de gentrificación.

Dimensión 2 (naturaleza): $\frac{\text { PobIDHalto }_{2017}}{\text { PobTOTAL }_{2017}}-\frac{\text { PobIDHalto }_{2011}}{\text { PobTOTAL }_{2011}}$

- Dimensión 3: Estructura del hogar. Las aportaciones que se han realizado al estudio de la gentrificación desde la geografía de la población y la demografía, han enfatizado la importancia de las transformaciones asociadas a las estructuras de lo hogares en los barrios que se han gentrificado (Hall et al., 1997). Estos cambios están vinculados a menudo a la llegada de población con nuevos comportamientos en la forma de convivencia, asociados a nuevos valores individuales y colectivos, y sintetizados en la teoría de la Segunda Transición Demográfica (Buzar et al., 2005). La elevada presencia de hogares unipersonales, de parejas sin hijos (y que viven fuera del matrimonio) o de hogares extensos no familiares (pisos compartidos) es más común en los barrios que se han gentrificado. De todos estos tipos de hogares, la lectura municipal del padrón sólo permite identificar a uno de ellos, los hogares unipersonales, que son los que proponemos incluir en nuestro indicador. Aquellos barrios en los que más ha crecido (entre 1-I-2011 y 1-I-2017) la proporción de los hogares unipersonales de adultos (16-64 años) puntúan más alto en el indicador de gentrificación.

Dimensión 3 (hogares unipersonales): $\frac{\operatorname{HogUNIP}_{2017}}{\operatorname{HogTOTAL}_{2017}}-\frac{\operatorname{HogUNIP}_{2011}}{\operatorname{HogTOTAL}_{2011}}$

- Dimensión 4: Atracción de la población cualificada. La presencia de población con estudios universitarios es un clásico en los indicadores de gentrificación (Atkinson et al., 2011). Esta es la primera dimensión que mediremos a través de los flujos migratorios y residenciales registrados en el padrón continuo, que además permite superar la clásica limitación de la lectura transversal del padrón continuo español, asociada con la dificultad de actualizar el nivel de instrucción de la población que no cambia de residencia. En esta dimensión medimos la proporción de llegadas a cada barrio (sea desde otros barrios de la ciudad o desde fuera del municipio) de personas con estudios universitarios respecto al total de llegadas (de la población de más de 25 años). Aquellos barrios en los que más ha crecido esta proporción (periodo 201617 en comparación con 2011-2012) puntúan más alto en el indicador de gentrificación.

Dimensión 4 (atracción cualificados) $\frac{\text { LlegUNIV }_{2016 \_17}}{\text { LlegTOTAL }_{2016 \_17}}-\frac{\text { LlegUNIV }_{2011_{112}}}{\text { LlegTOTAL }_{2011_{1} 12}}$

- Dimensión 5: Substitución de la población. La medición de los procesos de desplazamiento y expulsión de la población ha sido el gran talón de Aquiles en los estudios de la gentrificación (Newman y Wyly, 2006). Nosotros nos aproximamos a este aspecto incidiendo en los procesos de substitución de la población. Incorporamos al análisis las características de las personas que abandonan los barrios y las comparamos con las personas que llegan. En este caso, situamos el foco en la población con menos capacidad económica, de la que la literatura nos dice que debería ir en retroceso en los barrios gentrificados (Ley, 1986). Aquí tomamos como referencia a la población sin estudios universitarios, dada la fuerte relación entre clase 


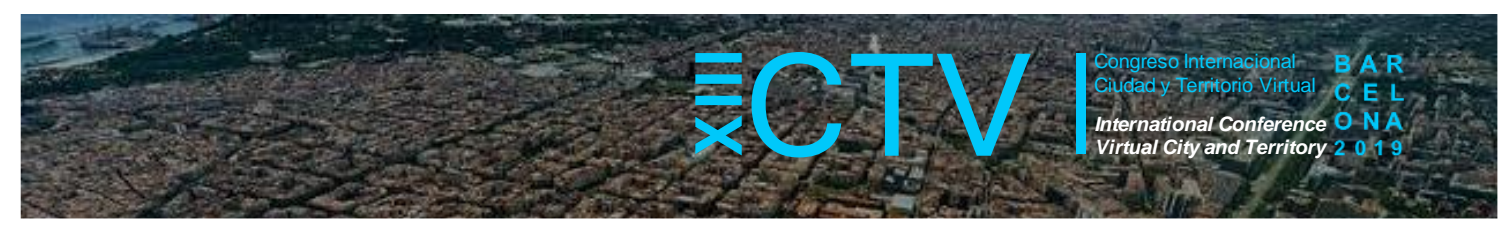

socioeconómica y nivel académico (Rubiales, 2017). Los barrios en los que las personas sin estudios universitarios están más representadas en los flujos de salida que en los de entrada están experimentando una paulatina substitución de la población a favor de los más educados. En el indicador utilizamos la proporción (y no los valores absolutos) de personas sin estudios universitarios (mayores de 25 años) en los flujos de salida y entrada, y calculamos una ratio que será más elevada cuanto mayor sea la presencia de personas sin estudios universitarios en los flujos de salida en comparación con los de entrada. Aquellos barrios en los que más ha crecido esta ratio (periodo 2016-17 en comparación con 2011-2012) puntúan más alto en el indicador de gentrificación.

Dimensión 5 (substitución): $\frac{\% \text { SalidNoUNIV } 2016 \_17}{\% \text { LlegNoUNIV }_{2016 \_17}}-\frac{\% \text { SalidNoUNIV }_{2011 \_12}}{\% \text { LlegNoUNIV }_{2011 \_12}}$

- Dimensión 6: Velocidad de los procesos de transformación. La composición de los flujos per se es insuficiente para medir los procesos de cambio sociodemográfico. Estas dinámicas están fuertemente determinadas por la intensidad de los flujos migratorios y residenciales, que repercuten directamente en la velocidad a la que se experimentan los cambios en la composición de la población. En el contexto urbano español, y sobre todo en las dos ciudades analizadas, existe una fuerte heterogeneidad espacial de la velocidad a la que se transforman los barrios, muy asociada al régimen de tenencia de la vivienda. En los barrios en los que avanza la gentrificación, la velocidad a la que se produce el proceso es alta y va en aumento (Rérat y Lees, 2011). El indicador elegido para sintetizar esta dimensión resulta de dividir los movimientos protagonizados por personas con estudios universitarios por el total de población (en ambos, de más de 25 años). Aquellos barrios en los que más ha crecido esta tasa (periodo 2016-17 en comparación con 2011-2012) puntúan más alto en el indicador de gentrificación.

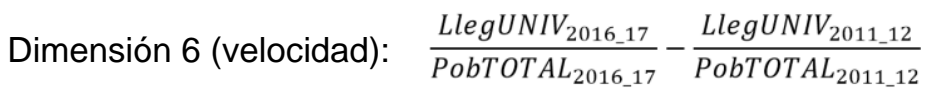

- Dimensión 7. Aumento del precio de la vivienda. Las dimensiones recogidas hasta ahora son de carácter sociodemográfico, pero es evidente el papel protagonista del mercado de la vivienda en los procesos de gentrificación (Smith, 1987). De todas las opciones posibles, decidimos utilizar aquí la evolución del precio de la vivienda como variable que sintetiza mejor las transformaciones del mercado. La disponibilidad de datos nos obliga a incluir tan sólo el precio de venta en el mercado de segunda mano si queremos construir la serie más larga posible a nivel de barrio. Pese a todo, sólo podemos llegar hasta el año 2013. Los ayuntamientos de las dos ciudades proporcionan datos a nivel de barrio basada en los informes de idealista ${ }^{2}$. Aquellos barrios en los que más ha aumentado de forma relativa (entre 2013 y $2017)$ el precio de la vivienda $\left(€ / \mathrm{m}^{2}\right)$ puntúan más alto en el indicador de gentrificación.

Dimensión 7 (precio vivienda): $\quad\left(\frac{€ / m^{2}{ }_{2017}-€ / m^{2}{ }_{2013}}{€ / m^{2}{ }_{2013}}\right) \times 100$

En cada una de las dimensiones no sólo medimos la diferencia del indicador utilizado entre los dos momentos analizados sino también el nivel que alcanza en 2017, ya que consideramos que es necesario introducir en el indicador la intensidad con la que se experimenta ese

\footnotetext{
${ }^{2}$ Existe algún vacío de información en los barrios más pequeños, a los que les hemos asignado los precios de los barrios limítrofes, o en su ausencia, los precios medios del distrito.
} 


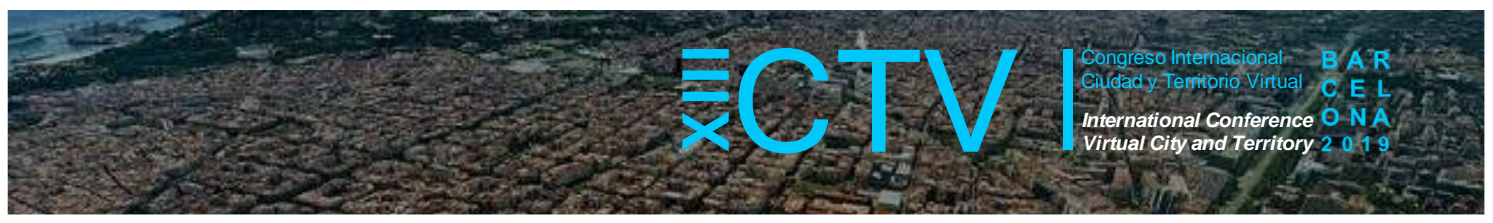

fenómeno. No obstante, como el objetivo principal del indicador final es el de medir las transformaciones de los procesos que se han experimentado entre los dos momentos de estudio, ponderamos doblemente el valor de la diferencia (por tanto, la diferencia temporal se pondera por $2 / 3$ y el nivel alcanzado en 2017 por 1/3). Los valores de cada una de las dimensiones se han normalizado en una escala de 0 a 1 (tanto los correspondientes a la diferencia como al nivel). Se ha realizado una interpolación lineal entre los 190 valores de los barrios tomando como extremo de la distribución el quinto valor más alto y el más bajo. Por tanto, los cinco valores más bajos de cada dimensión (tanto de diferencia como de nivel) puntúan 0 , y los cinco más altos puntúan $1^{3}$. Una vez realizada la ponderación de los valores referentes a la diferencia y al nivel alcanzado, volvemos a normalizar en una escala de 0 a 1 , esta vez tomando como extremos los valores mínimos y máximos de la distribución. El indicador final de gentrificación multiplica con el mismo peso el indicador de cada una de las dimensiones (por tanto, 1/7). En la Tabla 3 presentamos los valores extremos y la mediana de las diferencias y de los niveles alcanzados en las siete dimensiones contempladas.

Tabla 2. Valores extremos y mediana de las siete dimensiones del indicador de gentrificación

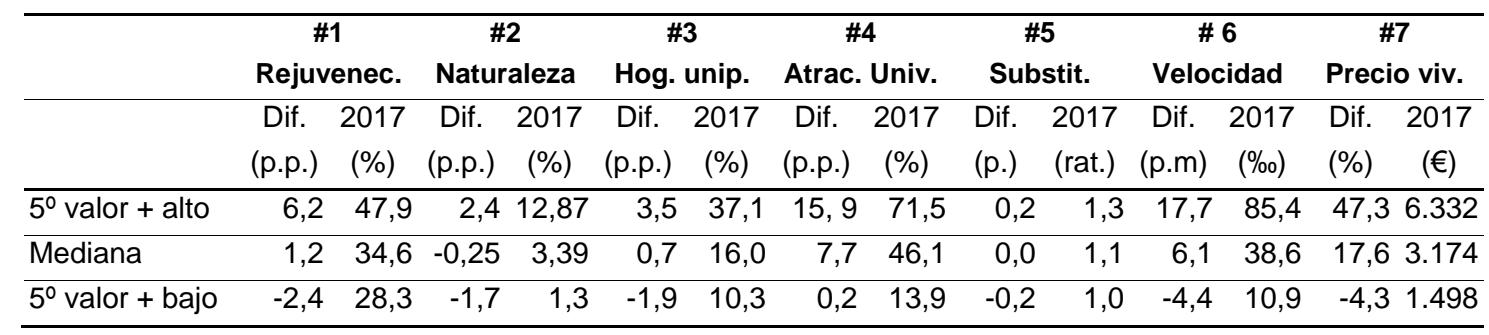

Fuente: elaboración propia. Notas: Dif=Diferencias entre el valor a final de periodo menos a comienzo de periodo; $2017=$ nivel en 2017; (p.p.)=puntos porcentuales; (rat.)=ratio; (p.)=puntos de diferencia en la ratio; (p.m.)=puntos por mil

Con el objetivo de analizar los principales atributos estadísticos del indicador hemos realizado un Análisis de Componentes Principales (ACP) con variación ortogonal varimax. El ACP es una de las técnicas más ampliamente usadas para reducir la dimensionalidad en una base de datos con diferentes variables, preservando la mayor variabilidad posible (Jolliffe et al., 2016). Para asegurarnos que se cumplían las condiciones para realizar el ACP hemos realizado una matriz de correlaciones de Spearman con las 7 dimensiones utilizadas en el índice. Además, hemos utilizado otros dos tests para evaluar la aplicabilidad del ACP: 1) test de Esfericidad de Barlett, con un valor de significación estadística del 5\%, y 2) test de Kaiser-Meyer-Olkin (KMO) para evaluar la adecuación muestral, tanto de la base de datos completa como de cada una de las dimensiones. Se ha estimado el porcentaje de contribución de cada dimensión con el primer componente del ACP y posteriormente se realizó un Análisis de Conglomerados Jerárquico (ACJ) con el método de Ward sobre el primer componente del ACP. El ACJ nos sirvió para analizar descriptivamente como podrían ser agrupados los barrios (Peña, 2002). Los análisis se han realizado tanto para las dos ciudades en conjunto, como por separado.

\section{Resultados: una aplicación a los municipios centrales de Barcelona y Madrid, 2011-2017}

A partir de las siete dimensiones presentadas generamos el indicador de gentrificación, que nos proporciona valores entre 0 y 1 para los 190 barrios incluidos en el análisis. El valor 1 lo habría obtenido aquel barrio que liderase cada una de las siete dimensiones contempladas y

\footnotetext{
${ }^{3}$ En el cálculo del Indicador de Gentrificación por separado para cada ciudad, se han utilizado dos valores extremos.
} 


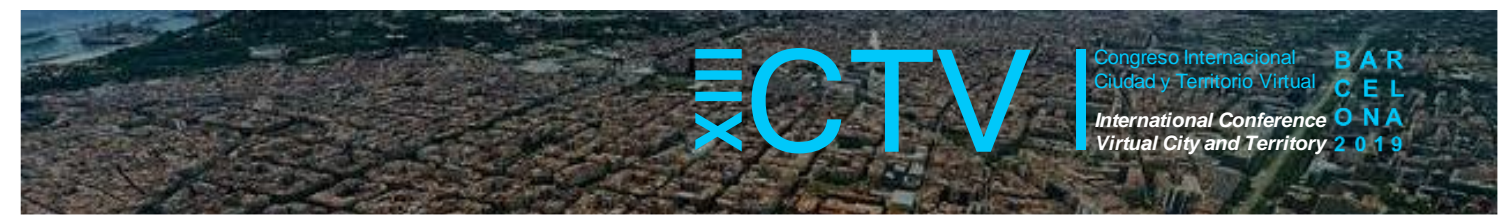

que corresponden a transformaciones asociadas a los procesos de gentrificación. Al contrario, el valor 0 lo habría obtenido el barrio a la cola de todas estas transformaciones. A partir del valor obtenido en el indicador de gentrificación, hemos construido cuatro categorías. Debido a que no existe ningún elemento teórico que nos permita delimitar el nivel exacto a partir del cual podemos hablar de gentrificación, proponemos considerar como barrios en proceso de gentrificación aquellos que se encuentran dentro del primer cuartil (1C) de la distribución. Dentro de este cuartil, consideramos oportuno diferenciar los barrios en dos categorías según el nivel de elitización de partida (en 2011). Entre las utilizadas, consideramos que una buena variable síntesis para captar este aspecto es la proporción de personas con estudios universitarios que llegó al barrio en 2011. El umbral que se ha considerado más apropiado es el del segundo quintil, es decir el que delimita los barrios con el $40 \%$ de valores más altos (en este caso, aquellos barrios en los que más del $43,25 \%$ de las personas que han llegado tenían estudios universitarios), a partir de ahora denominados "barrios previamente elitizados". Así pues, la primera categoría incluye los barrios en el primer cuartil del indicador de gentrificación y que previamente estaban elitizados. La segunda categoría incluye barrios del primer cuartil en el índice de gentrificación pero que no estaban previamente elitizados. Los barrios que se encuentran dentro del segundo cuartil los hemos agrupado en una tercera categoría, cuyos valores nos apuntan hacia una ligera tendencia gentrificadora entre 2011 y 2017 . Finalmente, la cuarta categoría incluye la mitad de barrios con el indicador de gentrificación más bajo.

Los resultados del indicador de gentrificación para cada uno de los barrios se presentan en la Figura 1 y en la Tabla 3, en la que también se incorporan los resultados que el indicador de obtendría si se analizasen las dos ciudades de forma independiente. Según este cálculo, Sant Pere, Santa Caterina i la Ribera, en el distrito barcelonés de Ciutat Vella, es el barrio que más transformaciones afines a los procesos de gentrificación ha experimentado entre 2011 y 2017 cuando analizamos conjuntamente Barcelona y Madrid. El indicador supera el 0,9, un valor que refleja una puntuación muy alta en todas las dimensiones analizadas. El barrio madrileño que puntúa más alto es Trafalgar, en el distrito de Chamberí, con un 0,81 , seguido muy de cerca por Universidad, en el distrito Centro. La Barceloneta y el Raval completan el listado de los cinco barrios que más transformaciones asociadas a los procesos de gentrificación han registrado entre 2011 y 2017 . El número de unidades en la categoría de barrios gentrificados es casi idéntica en Barcelona y en Madrid (23 y 25 respectivamente) y dibujan una gran zona central con ramificaciones específicas. En Barcelona, todos los barrios de los distritos de Ciutat Vella y del Eixample se circunscriben en esta categoría (en el caso del Eixample, los barrios ya estaban fuertemente elitizados en 2011). Todos los barrios alrededor de este sector central aparecen con valores elevados de gentrificación, algunos de los cuales ya estaban fuertemente elitizados en 2011, como la Vila de Gràcia o Sant Gervasi-Galvany, y otros muestran una transformación mucho más reciente, como el Poble Sec, Hostafrancs, el Poblenou y el Camp de l'Arpa del Clot. En Madrid, todos los barrios del distrito Centro alcanzan valores altos, una zona que se expande hacia el norte (la práctica totalidad de barrios del distrito de Chamberí, y los orientales de Tetuán) y hacia el noreste (barrios del distrito de Salamanca y los del sureste de Chamartín). Al sur, los barrios de Palos de Moguer y Chopera, en el distrito de Arganzuela, son los únicos que pertenecen a la categoría de barrios más gentrificados. Además, son los barrios que en 2011 presentaban una menor elitización, además de Embajadores, al sur del distrito Centro. Los barrios con los valores más bajos del indicador de gentrificación se sitúan en el distrito de Nou Barris y al norte del de Sant Andreu en Barcelona, y en el exterior de la M30 en Madrid. En este sector, únicamente los barrios de Puerta del Ángel, en Latina, de Comillas en Carabanchel, Quintana en Ciudad Lineal y Valdefuentes en Hortaleza, presentan valores que podrían estar manifestando la existencia de ligeras dinámicas asociadas a la 


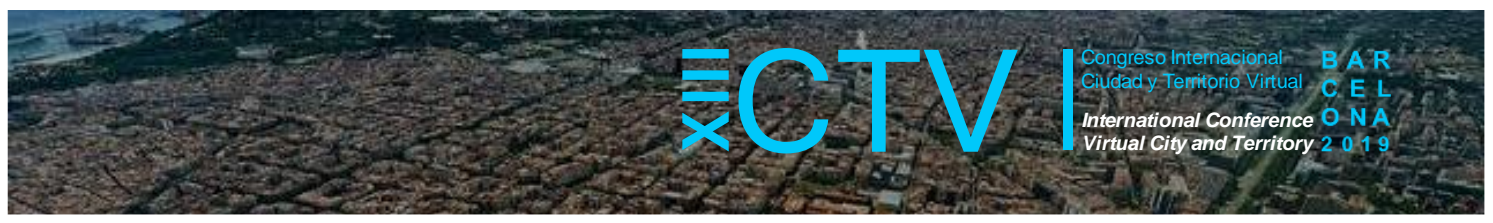

gentrificación. Por descontado, no podemos dejar de mencionar los posibles efectos de la diferente dimensión de Barcelona y Madrid. Aunque los barrios de ambas ciudades son de tamaño muy similar, lo que los hace plenamente comparables, la mayor superficie de Madrid, con muchos barrios alejados de las dinámicas sociodemográficas propias de los centros urbanos, explicaría que la gran mayoría de barrios con índices bajos se encuentren en la capital madrileña.

Figura 1. Resultados del indicador de gentrificación en Barcelona y Madrid, 2011-2017
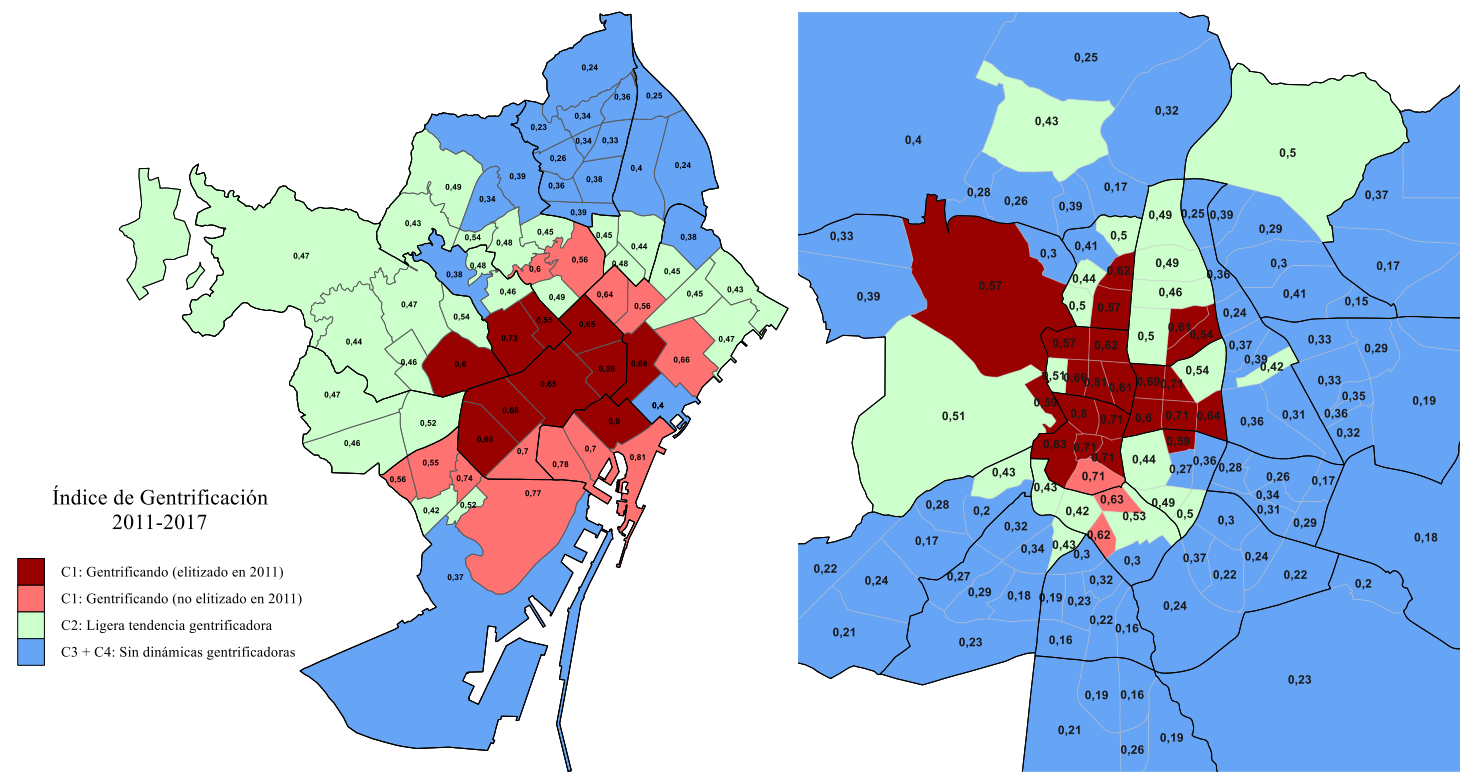

Fuente: Elaboración propia

Como hemos apuntado en el apartado metodológico, hemos realizado diferentes ejercicios para evaluar las características estadísticas del indicador propuesto. La correlación de las dimensiones ha mostrado que ninguna de ellas correlaciona más de 0,8 con otra. Si bien, la dimensión 3 (hogares unipersonales) no correlaciona con ninguna de las otras dimensiones excepto con la dimensión 6 (velocidad de los procesos de transformación) y la dimensión 1 (rejuvenecimiento). El test de esfericidad de Barllet es altamente significativo, lo que indicaría que hay homogeneidad de varianzas y por tanto se podía realizar el ACP. El test de KMO ha concluido con un valor de 0,73 para la muestra en su conjunto, lo que indica que las condiciones para hacer el ACP son adecuadas. Sin embargo, al analizar las dimensiones, la dimensión 3 (hogares unipersonales) presentó un valor de 0,43, que se consideraría como no adecuado. El primer componente del ACP explica un $57 \%$ de la varianza total, reflejando la naturaleza unidimensional del índice.

La dimensión que menos contribuye al índice global es la 3 (hogares unipersonales). Las tres dimensiones que más contribuyeron fueron: dimensión 4 (atracción de la población cualificada); dimensión 7 (aumento del precio de la vivienda); y dimensión 6 (velocidad de los procesos de transformación). En la Tabla 4 mostramos el porcentaje de contribución de las distintas dimensiones al índice de gentrificación estimado mediante PCA. La agrupación de barrios que resulta del análisis jerárquico es muy similar a la que hemos obtenido en nuestra propuesta. Los 48 barrios considerados gentrificados en nuestra propuesta (1 Cuartil) se agruparon en un mismo conglomerado. Sólo hubo 6 barrios que se agruparían como gentrificados en el análisis de conglomerados jerárquicos y que no aparecen como tales en nuestra propuesta. 


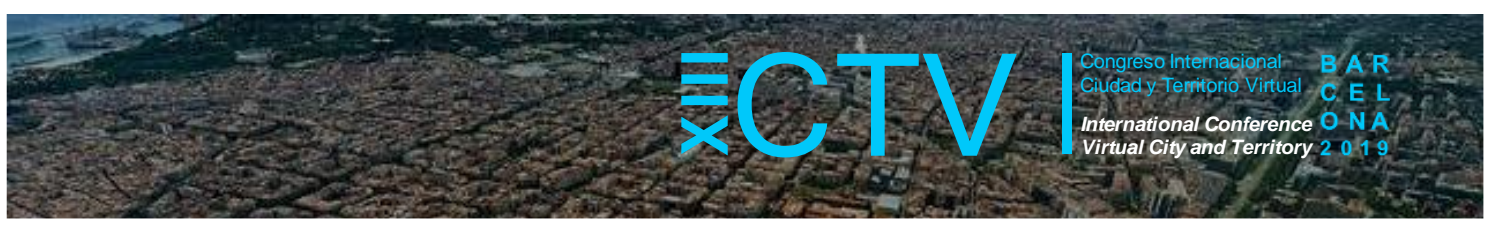

Tabla 3. Resultados del Indicador de Gentrificación

\begin{tabular}{|c|c|c|}
\hline & Ciudad & Distrito \\
\hline 1 & $\mathrm{BCN}$ & Ciutat Vella \\
\hline 2 & $\mathrm{BCN}$ & Ciutat Vella \\
\hline 3 & MAD & Chamberí \\
\hline 4 & MAD & Centro \\
\hline 5 & $\mathrm{BCN}$ & Ciutat Vella \\
\hline 6 & $\mathrm{BCN}$ & Sants-Montjuïc \\
\hline 7 & $\mathrm{BCN}$ & Sants-Montjuïc \\
\hline 8 & $\mathrm{BCN}$ & Gràcia \\
\hline 9 & MAD & Centro \\
\hline 10 & MAD & Centro \\
\hline 11 & MAD & Centro \\
\hline 12 & MAD & Salamanca \\
\hline 13 & MAD & Salamanca \\
\hline 14 & MAD & Centro \\
\hline 15 & $\mathrm{BCN}$ & Ciutat Vella \\
\hline 16 & $\mathrm{BCN}$ & Eixample \\
\hline 17 & MAD & Salamanca \\
\hline 18 & $\mathrm{BCN}$ & Sant Martí \\
\hline 19 & $\mathrm{BCN}$ & Eixample \\
\hline 20 & $\mathrm{BCN}$ & Sant Martí \\
\hline 21 & MAD & Chamberí \\
\hline 22 & $\mathrm{BCN}$ & Eixample \\
\hline 23 & $\mathrm{BCN}$ & Eixample \\
\hline 24 & $\mathrm{BCN}$ & Eixample \\
\hline 25 & $\mathrm{BCN}$ & Sant Martí \\
\hline 26 & MAD & Salamanca \\
\hline 27 & MAD & Centro \\
\hline 28 & MAD & Arganzuela \\
\hline 29 & MAD & Arganzuela \\
\hline 30 & MAD & Chamberí \\
\hline 31 & MAD & Tetuán \\
\hline 32 & MAD & Chamartín \\
\hline 33 & MAD & Chamberí \\
\hline 34 & $\mathrm{BCN}$ & Horta-Guinardó \\
\hline 35 & MAD & Salamanca \\
\hline 36 & $\mathrm{BCN}$ & Sarrià-Sant C \\
\hline 37 & MAD & Moncloa - Aravaca \\
\hline 38 & MAD & Retiro \\
\hline 39 & $\mathrm{BCN}$ & Eixample \\
\hline 40 & MAD & Moncloa - Aravaca \\
\hline 41 & MAD & Chamberi \\
\hline 42 & MAD & Tetuán \\
\hline 43 & $\mathrm{BCN}$ & Sants-Montjuïc \\
\hline 44 & $\mathrm{BCN}$ & Sant Martí \\
\hline 45 & $\mathrm{BCN}$ & Horta-Guinardó \\
\hline 46 & $\mathrm{BCN}$ & Sants-Montjü̈ \\
\hline 47 & $\mathrm{BCN}$ & Gràcia \\
\hline 48 & MAD & Chamartín \\
\hline 49 & MAD & Salamanca \\
\hline 50 & $\mathrm{BCN}$ & Sarrià-Sant Gervasi \\
\hline 51 & $\mathrm{BCN}$ & Horta-Guinardó \\
\hline 52 & MAD & Arganzuela \\
\hline 53 & $\mathrm{BCN}$ & Les Corts \\
\hline 54 & $\mathrm{BCN}$ & Sants-Montjuïc \\
\hline 55 & MAD & Moncloa - Aravaca \\
\hline 56 & MAD & Chamberí \\
\hline 57 & MAD & Tetuán \\
\hline 58 & MAD & Tetuán \\
\hline 59 & MAD & Chamartín \\
\hline 60 & MAD & Retiro \\
\hline 61 & MAD & Hortaleza \\
\hline 62 & MAD & Retiro \\
\hline 63 & $\mathrm{BCN}$ & Horta-Guinardó \\
\hline 64 & MAD & Chamartín \\
\hline 65 & $\mathrm{BCN}$ & Horta-Gu \\
\hline 66 & MAD & Chamartín \\
\hline 67 & $\mathrm{BCN}$ & Horta-Guinardó \\
\hline 68 & $\mathrm{BCN}$ & G \\
\hline 69 & $\mathrm{BCN}$ & Sant Andreu \\
\hline 70 & $\mathrm{BCN}$ & Les Corts \\
\hline 71 & $\mathrm{BCN}$ & Sant $M$ \\
\hline 72 & $\mathrm{BCN}$ & Sarrià-Sant Gervasi \\
\hline 73 & $\mathrm{BCN}$ & Sarrià-Sant Gervasi \\
\hline 74 & MAD & Chamartín \\
\hline 75 & $\mathrm{BCN}$ & Les Corts \\
\hline 76 & $\mathrm{BCN}$ & Sarrià-Sant \\
\hline 77 & $\mathrm{BCN}$ & Gràcia \\
\hline 78 & $\mathrm{BCN}$ & Martí \\
\hline 79 & $\mathrm{BCN}$ & uinardó \\
\hline 80 & $\mathrm{BCN}$ & s \\
\hline 81 & $\mathrm{BCN}$ & Sant Andreu \\
\hline 82 & MAD & Retiro \\
\hline 83 & $\mathrm{BCN}$ & $\mathrm{nt} A$ \\
\hline 84 & MAD & Te \\
\hline 85 & $\mathrm{BCN}$ & Sarrià-Sant Gervasi \\
\hline 86 & $\mathrm{BCN}$ & Horta-Guinardó \\
\hline 87 & MAD & \\
\hline 88 & $\mathrm{BCN}$ & \\
\hline 89 & MAD & Caraban \\
\hline 90 & MAD & Fuencarral - EI Pardo \\
\hline 91 & MAD & Arganzue \\
\hline 92 & $\mathrm{BCN}$ & \\
\hline 93 & MAD & \\
\hline 94 & MAD & Ciudad Lineal \\
\hline 95 & MAD & Barajas \\
\hline
\end{tabular}

Fuente: Elaboración propia. Notas: Categorías: 1=1C (elitizado en 2011); $2=1 \mathrm{C}$ (no elitizado en 2011); 3=2C; 4=3C+4C IG-BCN/MAD: resultados del ejercicio específico en cada ciudad. 


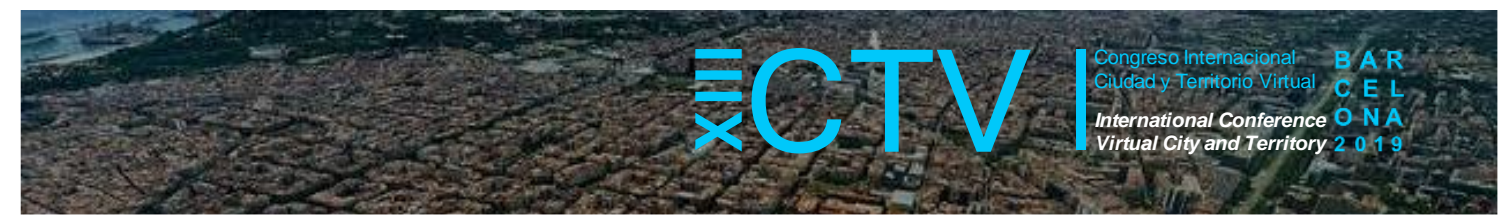

En el caso del indicador de gentrificación que hemos generado para el municipio de Barcelona de forma independiente, las dimensiones 1 y 2 se correlacionan en más de $0,8(0,83)$. Al igual que en el análisis conjunto, la dimensión 3 (hogares unipersonales) no correlaciona con ninguna de las otras dimensiones excepto con la dimensión 1 (rejuvenecimiento) y la dimensión 2 (naturaleza). El test de esfericidad de Barllet también ha sido altamente significativo. El test de $\mathrm{KMO}$ ha ofrecido un valor de 0,74 para la muestra en su conjunto. Del mismo modo que sucede en el análisis conjunto, la dimensión 3 (hogares unipersonales) presenta valores bajos $(0,58)$, pero mejores que en la muestra conjunta. El primer componente del ACP en Barcelona también explica un $57 \%$ de la varianza total, y las dimensiones que menos contribuyen son la dimensión 3 (hogares unipersonales), al igual que en el análisis conjunto, y la dimensión 5 (substitución de la población). A diferencia del análisis conjunto, la dimensión que más contribuye en el caso específico de Barcelona es la dimensión 2 (naturaleza de la población).

En el caso del indicador construido específicamente para Madrid, dos correlaciones excedieron el 0,8: a) la dimensión 4 con la dimensión 6 ; y b) la dimensión 1 con la dimensión 3 . A diferencia de la propuesta conjunta y la barcelonesa, no encontramos dimensiones que no presenten correlaciones bivariadas bajas entre ellas. El test de esfericidad de Barllet también es también altamente significativo. El test de $\mathrm{KMO}$ ha ofrecido un valor de 0,8 para la muestra en su conjunto. En general, en el caso de Madrid, todas las dimensiones presentan valores de KMO aceptables. El primer componente del ACP en Madrid explica un $61 \%$ de la varianza total. Al contrario que en el caso barcelonés, la dimensión que menos contribuye es la dimensión 2 (naturaleza de la población). En cambio, las que más contribuyen son la dimensión 6 (velocidad de sustitución) y la dimensión 7 (aumento del precio de la vivienda).

Tabla 4. Contribución porcentual de las dimensiones al índice

\begin{tabular}{lrrr}
\hline \multicolumn{1}{c}{ Dimensión } & \multicolumn{1}{c}{$\begin{array}{l}\text { Análisis } \\
\text { conjunto (\%) }\end{array}$} & Barcelona (\%) & \multicolumn{2}{l}{ Madrid (\%) } \\
\hline Dimensión 1 (rejuvenecimiento) & 16,50 & 18,99 & 16,15 \\
\hline Dimensión 2 (naturaleza) & 11,80 & 19,25 & 7,27 \\
\hline Dimensión 3 (hogares unipersonales) & 2,69 & 5,29 & 12,21 \\
\hline Dimensión 4 (atracción de la población cualificada) & 18,60 & 16,88 & 15,25 \\
\hline Dimensión 5 (substitución de la población) & 14,36 & 7,76 & 13,06 \\
\hline Dimensión 6 (velocidad de la transformación) & 17,68 & 18,29 & 18,57 \\
\hline Dimensión 7 (aumento del precio de la vivienda) & 18,37 & 13,54 & 17,40 \\
\hline
\end{tabular}

Fuente: Elaboración propia.

\section{Conclusiones}

Hacer frente a las causas y a los efectos derivados de los procesos de gentrificación es uno de los principales retos a los que están haciendo frente las dos ciudades más pobladas de España, Madrid y Barcelona. Como en muchas otras áreas urbanas del mundo, preocupa la transformación del espacio urbano y de la composición de la población (que favorece a los estratos más privilegiados) y por eso se han desarrollado una serie de políticas públicas para frenar esas dinámicas. Ahora bien, pese a esos avances en la esfera de la acción política, la gentrificación es todavía un proceso difícilmente cuantificable y por eso, su diagnosis ha sido tradicionalmente compleja. En este artículo queremos dar un paso en esa dirección y hemos propuesto un indicador para localizar territorialmente los espacios en los que se han experimentado esos procesos y para medir la intensidad con la que se han manifestado. Basándonos en aportaciones teóricas y experiencias cuantitativas internacionales hemos 


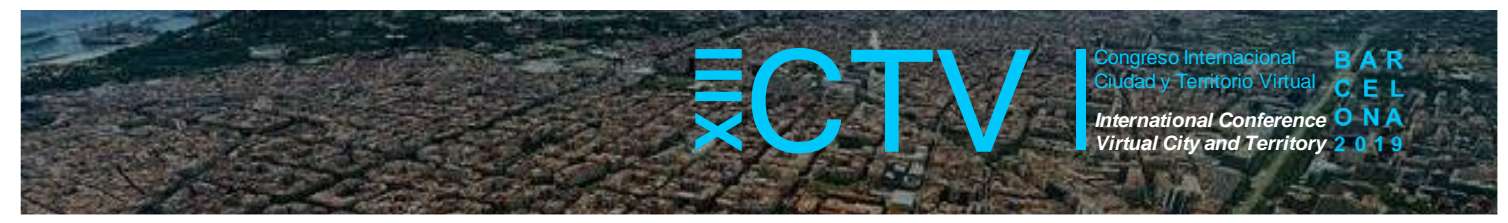

generado un indicador que engloba hasta siete dimensiones, de naturaleza esencialmente sociodemográfica. Los aspectos más innovadores de esta propuesta son la utilización de diversas fuentes de información, entre las que destaca el uso pionero de la composición de los flujos migratorios y residenciales, auténticos motores de los cambios poblacionales, y la comparabilidad de los resultados entre Barcelona y Madrid, al haber aplicado una metodología conjunta que incluye a todos los barrios de ambas ciudades.

Los resultados nos han permitido identificar qué barrios de ambas ciudades han experimentado con mayor intensidad cambios propios de los espacios gentrificados entre 2011 y 2017. En general, han sido los barrios céntricos de ambas ciudades, aunque no sólo los cascos históricos, los que han vivido mayores cambios en ese sentido. Más allá de esa zona central, el indicador calculado permite observar los ejes que han tomado los procesos de gentrificación durante este periodo, y vislumbrar hacia qué espacios está avanzando. En Barcelona ha sido clara la expansión hacia los ejes de Poble Sec, Sants-Hostafrancs, Poblenou y Camp de l'Arpa del Clot, mientras que en Madrid los ejes del norte (Chamberí) y del noreste (Salamanca) son los ámbitos donde los procesos afines a la gentrificación están más consolidados y en el del sur donde aparecen los espacios más emergentes (Palos de Moguer y Chopera, por ejemplo). La barrera de la M30 continúa inquebrantable, aunque aparecen niveles relativamente altos en algún barrio como Comillas, en Carabanchel. Por otra parte, los tests estadísticos utilizados avalan la fortaleza de la aplicación de nuestro modelo teórico, pero también revelan que existen dos dimensiones que funcionan de forma específica en cada ciudad. Por un lado, la vinculación de los procesos de gentrificación con el aumento de población nacida en países de ingresos altos tiene una especial validez en el caso de Barcelona, mientras que en Madrid esta relación es mucho más diluida. En cambio, la asociación entre el aumento de los hogares unipersonales de adultos con los espacios gentrificados es más fuerte en Madrid que en Barcelona, una situación que podría estar explicada por el freno que el aumento del precio de la vivienda ha representado para la expansión de este tipo de hogares en la capital catalana y que ha favorecido la rápida expansión de otras formas de convivencia, como los pisos compartidos.

Finalmente, creemos que el indicador que hemos propuesto posee una gran potencialidad como herramienta para la toma de decisiones que garanticen una mayor justicia urbana y social. Además, consideramos que puede tener un largo recorrido para el diagnóstico de estos procesos. Por un lado, la versatilidad del indicador le permitiría integrar fácilmente otras dimensiones también asociadas a la gentrificación y que van más allá de las sociodemográficas. Indicadores sobre transformaciones en la actividad comercial, en el desarrollo urbanístico e inmobiliario o en la actividad turística podrían ser incluidos. Por el otro, el indicador podría ser una gran herramienta de monitoreo, casi a tiempo real, de los procesos de gentrificación, un aspecto fundamental para la toma de decisiones. Además, a medida que avance el tiempo y la serie de datos se alimente de más años observados, se podrá comparar la intensidad de los procesos en diferentes momentos y diseccionar la forma en la interaccionan en el tiempo y en el espacio cada una de las dimensiones del indicador de gentrificación.

Agradecimientos: Esta investigación se ha realizado en el marco de diversos proyectos de investigación: Programa Talent de la Universitat Autònoma de Barcelona; Proyecto GLOBFAM del plan I+D+I del Ministerio de Ciencia, Innovación y Universidad (es) (RTI2018-096730-B100); Proyecto EQUALIZED de la European Research Council (ERC-2014-StG-637768). 


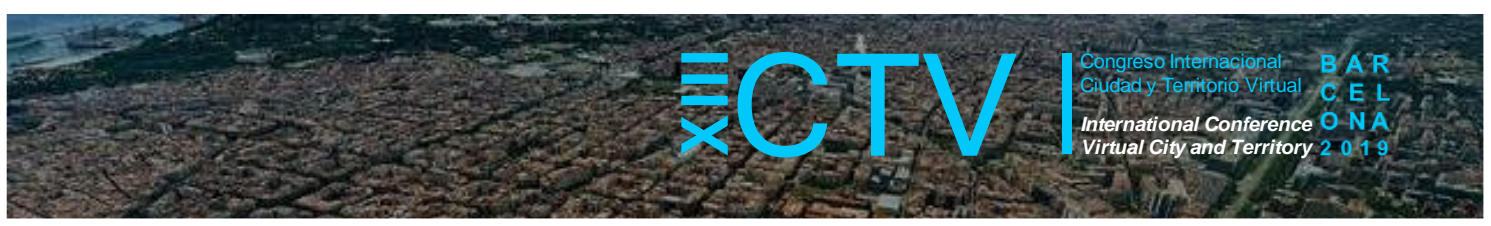

Contribuciones de los autores: Todos los autores han contribuido en la concepción del artículo y del indicador, así como en la revisión del manuscrito final. El primer autor ha desarrollado la construcción del indicador y el análisis de datos, el segundo y el tercer han elaborado conjuntamente el marco teórico y la cuarta y el quinto han realizado el análisis de componentes principales y el análisis jerárquico de conglomerados.

Conflicto de Intereses: Los autores declaran que no hay conflicto de intereses.

\section{Bibliografía}

Albet, A. y Benach, N. (2018). Gentrification as a global strategy. Neil Smith and beyond. Londres: Routledge.

Atkinson, R. (2000). Measuring Gentrification and Displacement in Greater London. Urban Studies, 37:1, 149-165.

Atkinson, R. y Bridge, G. (Eds.) (2005). Gentrification in a Global Context: The New Urban Colonialism. Londres: Routledge.

Atkinson, R., Wulff, M., Reynolds, M. y Spinney, A. (2011). Gentrification and displacement: the household impacts of neighbourhood change. AHURI Final Report, 160, 1-89.

Antón-Alonso, F.; Porcel, S. y Cruz, I. (2018). Factors contextuals associats als processos de gentrificació de l'àrea metropolitana de Barcelona. Papers: Regió Metropolitana de Barcelona, 60, 64-79.

Bailey, N. y Minton. J. (2018). The suburbanisation of poverty in British cities, 2004-16: extent, processes and nature. Urban Geography, 39, no. 6: 892-915.

Bauman, Z. (1998). La globalización. Consecuencias humanas. México: FCE.

Bayona, J. y López-Gay, A. (2011). Concentración, segregación y movilidad residencial de los extranjeros en Barcelona. Documents d'Anàlisi Geogràfica, 57(3), 381-412.

Bianco, F.; Chapple, K.; Kleiman, N.; Sobolevsky, S.; Chermesh, D.; Xi, H.; Rodríguez, G. y Hambardzumyan, R. (2018). Map of Gentrification and Displacement for The Greater New York. Recuperado de http://www.udpny.org/static/media/report.8f3f1564.pdf

Bondi, L. (1999). Gender, class, and gentrification: enriching the debate. Environment and Planning D: Society and Space, 17(3), 261-282.

Buzar, S., Ogden, P. E. y Hall, R. (2005). Households matter: the quiet demography of urban transformation. Progress in Human Geography, 29(4), 413-436.

Ding, L.; Hwang, J. y Divringi, E. (2016). Gentrification and residential mobility in Philadelphia. Regional Science and Urban Economics, 61, 38-51. 


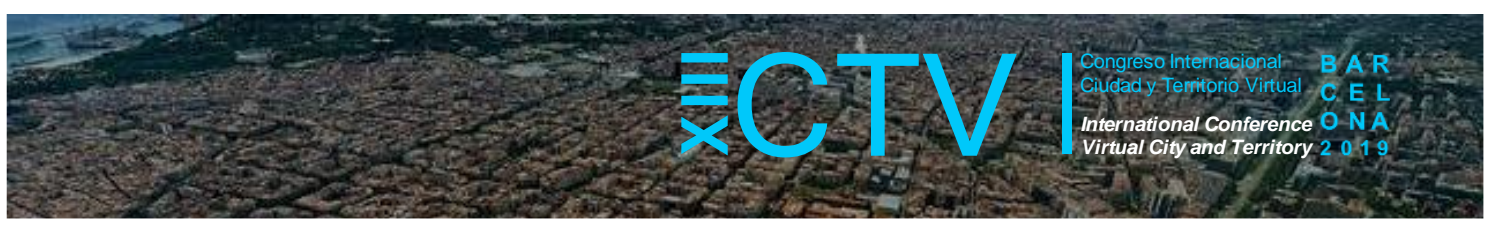

Donat, Carles (2018). La gentrificació i el problema de l'habitatge a Barcelona. Papers: Regió Metropolitana de Barcelona, 60, 114-129.

Freeman, L. (2005). Displacement or succession? Residential mobility in gentrifying neighborhoods. Urban Affairs Review, 40(4), 463-491.

Gale, D. (1985). Demographic research on gentrification and displacement. Journal of Planning Literature, 1, 14-29.

Glass, R. (1964). Introduction: aspects of change. In London: Aspects of Change. Centre for Urban Studies, Londres: MacKibbon and Kee.

Hall, R.; Ogden, P. E. y Hill, C. (1997). The pattern and structure of one-person households in England and Wales and France. International Journal of Population Geography, 3(2), 161-181.

Hochstenbach, C. y Musterd, S. (2017). Gentrification and the suburbanization of poverty: changing urban geographies through boom and bust periods. Urban Geography, 39 (1), 26-53. Hutton, T. A. (2009). The new economy of the inner city: restructuring, regeneration and dislocation in the 21st century metropolis. Nueva York: Routledge.

I-team. (2016). The Los Angeles Indices of Neighborhood Change. Recuperado de http://lahub.maps.arcgis.com/apps/MapJournal/index.html?appid=e3f1806c03fc4d0cbc7145aad $\underline{8 \mathrm{e} 523 \mathrm{~d} 9}$

Jolliffe, I. T. y Cadima, J. (2016). Principal component analysis: a review and recent developments. Phil. Trans. R. Soc. A., 374 (2065), 20150202.

Lees, L. (2012). The geography of gentrification: Thinking through comparative urbanism. Progress in Human Geography, 36(2), 155-171.

Lees, L.; Slater, T. y Wyly, E. (2008). Gentrification. Nueva York: Routledge.

Lees, L.; Bang Shin, H. y López-Morales, E. (2016). Planetary gentrification. Bristol: Policy Press.

Ley, D. (1986). Alternative explanations for inner-city gentrification: A Canadian assessment. Annals of the association of american geographers, 76(4), 521-535.

López-Gay, A. (2016a). Barcelona's got talent: migration, residential change and socioeconomic polarisation. Perspectives Demogràfiques, 3, 1-4.

López Gay, A. (2016b). El advenimiento de las regiones metropolitanas maduras en España: retos demográficos y socioeconómicos en un nuevo contexto urbano. Panorama Social, 23, 179-196. 


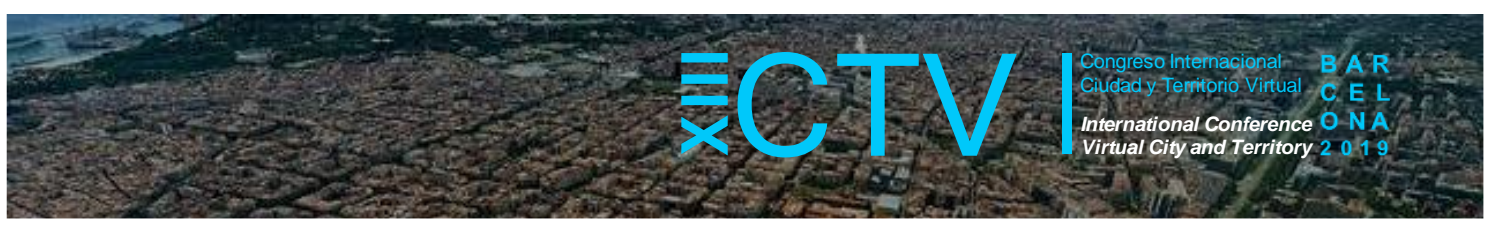

López-Gay, A. (2018). Cambio en la composición social y gentrificación en Barcelona: una mirada a través de los flujos migratorios y residenciales. Papers: Regió Metropolitana de Barcelona, 60, 80-93.

Martin, I. W. y Beck, K. (2018). Gentrification, property tax limitation, and displacement. Urban Affairs Review, 54(1), 33-73.

Martínez García, S. (2013). Estructura social y desigualdad en España. Madrid: Catarata.

Moos, M., Revington, N., Wilkin, T. y Andrey, J. (2019). The knowledge economy city: Gentrification, studentification and youthification, and their connections to universities. Urban Studies, 56(6), 1075-1092.

Musterd, S. (2006). Segregation, urban space and the resurgent city. Urban Studies, 43(8), $1325-1340$.

Newman, K. y Wyly, E. K. (2006). The right to stay put, revisited: Gentrification and resistance to displacement in New York City. Urban studies, 43(1), 23-57.

Ogden, P. E. y Schnoebelen, F. (2005). The rise of the small household: demographic change and household structure in Paris. Population, space and place, 11(4), 251-268.

Peña, D. (2002). Análisis de datos multivariantes. Madrid: McGraw-Hill Interamericana de España S.L.

Porcel, S.; Navarro-Varas, L.; Antón-Alonso, F. y Cruz, I. (2018). La suburbanització de la pobresa com a efecte metropolità de la gentrificació: el cas de Barcelona. Papers: Regió Metropolitana de Barcelona, 60, 95-113.

Riera, J. (2018). L'índex de gentrificació de Barcelona. Barcelona: Edicions Els Llums.

Rérat, P. y Lees, L. (2011). Spatial capital, gentrification and mobility: evidence from Swiss core cities. Transactions of the Institute of British Geographers, 36(1), 126-142.

Rubiales Pérez, M. (2016). Territorio y crisis. Impacto territorial de la crisis económica en las regiones metropolitanas de Madrid y Barcelona. Scripta Nova, vol. XX, 549.

Rubiales, M. (2017). Patrones socioterritoriales de las clases altas en las regiones metropolitanas de Barcelona y Madrid (2001 - 2015) (tesis doctoral). Universitat de Barcelona, Barcelona.

Rubiales, M.; Bayona, J. y Pujadas, I. (2012). Patrones espaciales de la segregación residencial en la Región Metropolitana de Barcelona: Pautas de segregación voluntaria de los grupos altos. Scripta Nova, Revista Electrónica de Geografía y Ciencias Sociales, XVI, 423.

Sarasa, S.; Navarro, L. y Porcel, S. (2017). Escenari post-crisi i estructura social metropolitana: vulnerabilitats i cobertura de necessitats des d'una mirada de clase. Bellaterra: IERMB. 


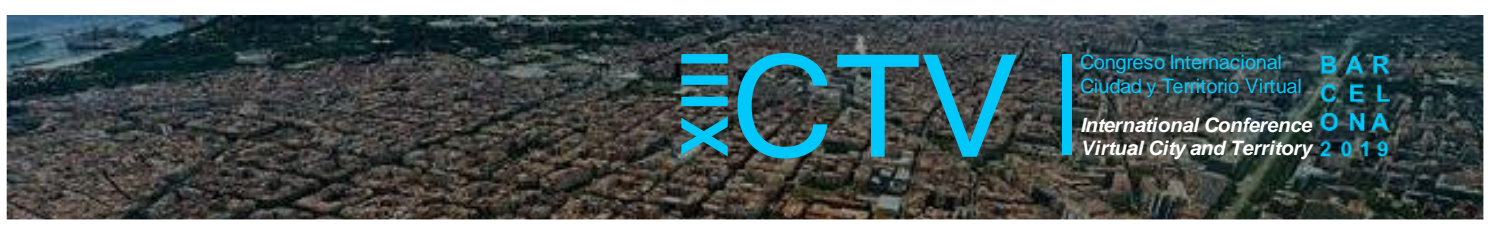

Sassen, S. (1991). The global city. Nueva York: Princeton University Press.

Sassen, S. (2018). Cities in a world economy. Thousand Oaks: SAGE Publications.

Smith, N. (1987) Gentrification and the Rent Gap. Annals of the Association of American Geographers, 77:3, 462-465.

Sorando, D. y Ardura, A. (2018). Procesos y dinámicas de gentrificación en las ciudades españolas. Papers: Regió Metropolitana de Barcelona, 60, 34-47.

Sorando, D. y Leal, J. (2019). Distantes y desiguales: el declive de la mezcla social en Barcelona y Madrid. Revista Española de Investigaciones Sociológicas, 167, 125-148.

Verma, P.; Rinzler, D.; Zuk, M.; DaSilva, M. y Kaplan E. (2018). Rising Housing Costs and ReSegregation in San Francisco. Recuperado de https://www.urbandisplacement.org/sites/default/files/images/sf final.pdf 